\title{
Inpatient Language Barriers: An Old Problem in Need of Novel Solutions
}

\author{
K Casey Lion, MD, MPH ${ }^{1,2 \star}$, Lisa Ross DeCamp, MD, MSPH ${ }^{3}$
}

${ }^{1}$ Department of Pediatrics, University of Washington School of Medicine, Seattle, Washington; ${ }^{2}$ Center for Child Health, Behavior and Development, Seattle Children's Research Institute, Seattle, Washington; ${ }^{3}$ Department of Pediatrics, University of Colorado School of Medicine and Children's Hospital Colorado, Aurora, Colorado

rer

he 25 million people in the United States with limited English proficiency (LEP), which is defined as speaking English less than "very well", are at increased risk for healthcare disparities that result in preventable harm and poor patient experiences compared with English-proficient patients. ${ }^{1,2}$ The use of trained professional interpreters is associated with improved communication, healthcare outcomes, safety, and experiences for LEP patients. ${ }^{3}$ However, underuse of professional interpreters remains common. ${ }^{4}$ Healthcare staff frequently use family members, friends, or minor children as interpreters or try to "get by" with the patient's limited English skills or staff's limited non-English skills. ${ }^{5}$ These practices regularly compromise patient safety and quality for LEP patients and their families.

In the article "Inpatient Communication Barriers and Drivers when Caring for Limited English Proficiency Children," Dr. Choe and colleagues approach the problem of interpreter underuse by studying the barriers and facilitators that exist at their children's hospital. ${ }^{6}$ The group conducted four sessions using Group Level Assessment, a structured, interactive approach to understanding a problem and identifying potential solutions. Sixty-four pediatric hospitalists and residents, bedside nurses, and staff interpreters participated. Participants identified four primary barriers to communicating effectively with LEP families: difficulty accessing interpreter services, uncertainty in communicating with LEP families, unclear roles and expectations of different team members, and unmet expectations related to family engagement. They also identified four drivers of effective communication: collaborative problem-solving between providers and interpreters, greater attention to cultural context, practicing empathy for patients and families, and using family centered communication strategies.

This study reinforces that myriad challenges remain in accessing and using an interpreter. The barriers identified fall into two major categories: systems for accessing interpretation and communication involving an interpreter. Both ultimately must be addressed to achieve equitable communication for LEP patients/families. As interpreter use is contingent upon access, optimizing delivery systems is an essential foundation. At this study site, key barriers were the opaque scheduling processes

*Corresponding Author: K. Casey Lion, MD, MPH; E-mail: casey.lion@seattlechildrens.org; Telephone: 206-884-1049

Received: May 31, 2019; Accepted: June 3, 2019

(c) 2019 Society of Hospital Medicine DOI 10.12788/jhm.3260 and inconsistent access to and unfamiliarity with interpreter-related technology (eg, for telephone or video interpretation). These barriers are likely generalizable to many other hospitals. Priority should be given to developing transparent, consistent, and reliable processes for interpreter access. Interventions to improve interpreter access, such as one-touch interpreter telephones at every hospital bedside, have been more successful in improving interpreter use than provider education or regulatory mandates. ${ }^{4}$

The challenges identified around communicating with LEP families via interpreter are also likely generalizable. In the current study, participants described a clear tension around the interpreters' optimal role, in which the care team might want the interpreter to intervene or participate in the discussion more, while interpreter standards require that they remain a neutral conduit for information. This neutral-party approach, when taken to the extreme, can limit the bidirectional communication between clinical teams and interpreters necessary to address communication challenges. Fostering collaborative problem-solving between interpreters and clinicians, in both formal and informal settings, is critically needed to improve the quality of communication during encounters. In addition to the proposed presession meeting between the clinician and interpreter, incorporating a debriefing after an interpreter-mediated encounter could offer an opportunity for bidirectional feedback. Unfortunately, interpreter scheduling constraints, fueled by the lack of reimbursement for interpretation in most states, frequently limit the feasibility of such proposals.

Participating providers also reported decreased engagement with LEP families and that they spent less time with them. These observations also merit attention if we are to achieve equitable outcomes for LEP patients. A conversation via interpreter requires more time for the same content, given the time needed to interpret the message. The fact that participants reported spending less time with LEP families means that less communication occurs with those families, compared with others. There are well-established links between good communication and improved clinical outcomes, including everything from decreased glycosylated hemoglobin levels to lower inpatient narcotic use. ${ }^{7}$ Thus, it is not surprising that patients with fewer opportunities to communicate fully have worse clinical outcomes. ${ }^{8}$ Addressing this will require changing hospital culture and provider expectations. Healthcare systems could support this effort with interventions such as decreased nursing assignments, longer allocated rounding times, longer outpatient 
clinic visits, and additional "points" in resident patient caps, if they exist, for LEP patients. Such steps would be an important investment in improving outcomes and decreasing costs for these vulnerable patients.

For all the barriers identified by Choe and colleagues, solutions are needed. Some may be generalizable, some may be location-specific, and most will be somewhere in between, requiring context-specific tailoring. We recommend a quality improvement ( $\mathrm{Ql}$ ) approach, as the evidence-based best practice for communicating with LEP patients and families is wellknown, but the gap is in delivering care that meets that standard. Leveraging the growing $\mathrm{Q}$ l expertise at many institutions to devise approaches that go beyond provider education to change the systems and culture around communicating with LEP patients holds our best promise for improving the safety and effectiveness of care for this population.

Disclosures: The authors have no financial relationships relevant to this article to disclose nor do they have any conflicts of interest relevant to this article to disclose.

Funding: Dr. Lion's time was supported by the Eunice Kennedy Shriver National Institute of Child Health and Human Development, grant K23 HD078507 (PI Lion).

\section{References}

1. Divi C, Koss RG, Schmaltz SP, Loeb JM. Language proficiency and adverse events in US hospitals: A pilot study. Int J Qual Heal Care. 2007;19(2):60-67. https://doi.org/10.1093/intqhc/mzl069

2. Yeheskel A, Rawal S. Exploring the "patient experience" of individuals with limited English proficiency: A scoping review. J Immigr Minor Heal. 2018. https://doi.org/10.1007/s10903-018-0816-4.

3. Karliner LS, Jacobs EA, Chen AH, Mutha S. Do professional interpreters improve clinical care for patients with limited English proficiency? A systematic review of the literature. Heal Serv Res. 2007;42(2):727-754. https://doi. org/10.1111/j.1475-6773.2006.00629.x.

4. Taira BR, Kim K, Mody N. Hospital and health system-level interventions to improve care for limited English proficiency patients: A systematic review. Jt Comm J Qual Patient Saf. 2019. https://doi.org/10.1016/j.jcjq.2019.02.005.

5. Diamond LC, Schenker Y, Curry L, Bradley EH, Fernandez A. Getting by: Underuse of interpreters by resident physicians. J Gen Intern Med. 2009;24(2):256-262. https://doi.org/10.1007/s11606-008-0875-7.

6. Choe A, Unaka N, Schondelmeyer A, Raglin Bignall W, Vilvens H, Thomson J. Inpatient communication barriers and drivers when caring for limited English proficiency children. J Hosp Med. 2019;14(10):607-613. https://doi. org/10.12788/jhm.3240.

7. Stewart MA. Effective physician-patient communication and health outcomes: A review. CMAJ. 1995;152(9):1423-1433.

8. Pérez-Stable EJ, El-Toukhy S. Communicating with diverse patients: How patient and clinician factors affect disparities. Patient Educ Couns. 2018;101(12):2186-2194. https://doi.org/10.1016/j.pec.2018.08.021. 